\title{
Flora da Bahia: Astraea (Euphorbiaceae)
}

\author{
Otávio Luis Marques da Silva ${ }^{1^{*}}$, Daniela Santos Carneiro Torres ${ }^{2, a} \&$ Inês Cordeiro ${ }^{1, b}$ \\ ${ }^{1}$ Programa de Pós-graduação em Biodiversidade Vegetal e Meio Ambiente, Instituto de Botânica, Núcleo de \\ Pesquisa Curadoria do Herbário SP, São Paulo, Brasil. \\ ${ }^{2}$ Programa de Pós-graduação em Botânica, Departamento de Ciências Biológicas, Universidade Estadual de Feira \\ de Santana, Feira de Santana, Bahia, Brasil.
}

\begin{abstract}
Resumo - Apresentamos aqui o tratamento taxonômico do gênero Astraea (Euphorbiaeae) na Bahia. O estado, junto com Minas Gerais, possui a maior diversidade de Astraea, com sete espécies reconhecidas: A. digitata, A. gracilis, A. klotzschii, A. paulina, A. praetervisa, A. subcomosa e A. surinamensis. O tratamento inclui chave de identificação, descrições, fotografias e mapas de distribuição, além de comentários relativos à distribuição geográfica, habitat e fenologia.
\end{abstract}

Palavras-chave adicionais: Brasil, Crotoneae, Taxonomia.

\begin{abstract}
Flora of Bahia: Astraea (Euphorbiaceae)) - This work presents the taxonomic treatment of the genus Astraea in Bahia. The state, along with Minas Gerais, has the highest diversity of Astraea, with seven species recognized: A. digitata, A. gracilis, A. klotzschii, A. paulina, A. praetervisa, A. subcomosa and A. surinamensis. The treatment includes an identification key, descriptions, photographs and distribution maps, besides comments on geographical distribution, habitat and phenology.
\end{abstract}

Additional key words: Brazil, Crotoneae, Taxonomy.

Euphorbiaceae possui cerca de 6.750 espécies e 200 gêneros, e distribuição quase cosmopolita, apesar de mais diversa ao longo dos trópicos (Webster 2014). A família é muito variada morfologicamente, mas características diagnósticas em campo incluem a comum presença de látex, folhas alternas com estípulas, flores em geral diminutas e sempre unissexuadas, as pistiladas com ovário súpero, trilocular e com apenas um óvulo por lóculo do ovário, além dos frutos do tipo cápsula com deiscência tanto septicida quanto loculicida, conhecidos popularmente como tricocas, com uma semente por lóculo, que pode apresentar carúncula (Webster 2014). No Brasil, Euphorbiaceae está entre as 10 famílias mais representativas, contando com 970 espécies em 64 gêneros, e elevado grau de endemismo (BFG 2015; Flora do Brasil 2020, em construção). Já para a flora da Bahia, são registradas cerca de 320 espécies de Euphorbiaceae, e a família têm sido abordada em diversas floras locais (e.g., Cordeiro 1995; Carneiro-Torres et al. 2014; Hurbath et al. 2016), porém o tratamento para Euphorbia L. foi único publicado dentro do projeto Flora da Bahia (Carneiro-Torres et al. 2017).

A família está atualmente dividida em 4 subfamílias (Cheilosoideae, Acalyphoideae, Crotonoideae e Euphorbioideae), compreendendo 29 tribos (Webster 2014). Entre as tribos, o grupo de maior destaque em toda região Neotropical é Crotoneae, na qual está o gigante Croton L., representado no Brasil por cerca de

\footnotetext{
*Autor para correspondência: otaviolmarques@gmail.com;

adscarneiro@hotmail.com; bisandona@uol.br

Editor responsável: Alessandro Rapini

Submetido: 6 maio 2020; aceito: 26 jun. 2020

Publicação elestrônica: 17 jul. 2020; versão final: 23 jul. 2020
}

300 espécies (Flora do Brasil 2020, em construção), além de Acidocroton Griseb., Astraea Klotzsch, Brasiliocroton P.E. Berry \& Cordeiro, Sagotia Baill e Sandwithia Lanj. (Wurdack et al. 2005; Riina et al. 2014; Webster 2014; Silva et al. 2020). As espécies de Astraea, foco deste trabalho, podem ser reconhecidas entre os gêneros de Euphorbiaceae pelo hábito herbáceo a arbustivo; folhas alternas, frequentemente partidas, sem nectários na base da lâmina (mas com um aglomerado de coléteres nesta mesma posição); inflorescências espiciformes; flores pistiladas com lobos do cálice acrescente no fruto e portando coléteres ao longo da margem, e estiletes cilíndricos e multífidos; flores estaminadas com pétalas portando tricomas moniliformes ao longo da porção basal das margens e estames inflexos no botão; e frutos geralmente lustrosos, com sementes tetragonais, arredondadas apenas em A. cincta (Müll.Arg.) Caruzo \& Cordeiro, e com carúncula (Silva \& Cordeiro 2020).

\section{Astraea Klotzsch}

Ervas ou subarbustos, monoicos, glabros ou com indumento variado, composto por tricomas simples, estrelados ou estrelado-porrectos, látex incolor ou esbranquiçado, geralmente não abundante. Folhas simples, alternas, inteiras ou 2-5-partidas, às vezes ambos os tipos em um mesmo indivíduo, margens serreadas a crenadas; estípulas geralmente subuladas, inteiras ou partidas. Inflorescências do tipo tirso especiforme, terminais; címulas proximais unissexuadas (apenas pistiladas) ou bissexuadas, címulas distais exclusivamente estaminadas, com 1-6 flores cada; brácteas subuladas a lineares. Flores estaminadas diclamídeas, dispostas em címulas com 1- 
6 flores; cálice gamossépalo, lobos 5, unidos apenas na base; corola dialipétala, pétalas 5 , com indumento denso de tricomas moniliformes ao longo da porção basal da margem; receptáculo glabro ou piloso; nectários 5, opostos às sépalas; estames 11-15, filetes livres, encurvados no botão, anteras 2-tecas, basifixas, extrorsas, tecas divergentes, deiscência horizontal. Flores pistiladas monoclamídeas; cálice gamossépalo, lobos 5-7, unidos apenas na base, acrescentes no fruto, com coléteres ao longo da margem; ovário 3-locular, com um óvulo por lóculo, estiletes 3, 6-8-ramificados, unidos na base. Frutos cápsulas de deiscência septicidaloculicida, geralmente lustrosas; sementes geralmente tetragonais em secção transversal, verrucosas, carunculadas.

Astraea é um gênero monofilético (Silva et al. 2020) com 13 espécies, encontrado ao longo de toda a região neotropical, e também no Velho Mundo, mas então apenas representado por espécies ruderais e invasoras: A. lobata (L.) Klotzsch, A. surinamensis (Miq.) O.L.M.Silva \& Cordeiro e A. trilobata (Forssk.) O.L.M.Silva \& Cordeiro (Silva \& Cordeiro 2020). A maior diversidade do gênero se concentra no leste do Brasil, sendo Bahia e Minas Gerais os estados com o maior número de espécies (Flora do Brasil 2020, em construção). Na Bahia, foram registradas sete espécies de Astraea, que ocorrem em todos os domínios fitogeográficos do estado, mas com maior diversidade na Mata Atlântica. Estas espécies podem ser encontradas em ambientes antropizados, campos rupestres, bordas de florestas úmidas e estacionais, matas de galeria ou restingas. Ilustrações e/ou imagens de campo de todas as espécies tratadas aqui também podem ser encontradas em Silva \& Cordeiro (2020).

\section{Chave para as espécies}

1. Estípulas glanduliformes 5. A. praetervisa

1'. Estípulas subuladas ou deltoides.

2. Indumento dos ramos jovens composto por tricomas estrelado-porrectos com raio central muito maior que os laterais (até 3-4 mm compr.)

7. A. surinamensis

2 '. Indumento dos ramos jovens composto por tricomas simples, estrelados ou, se estreladoporrectos, com raio central não muito maior que os laterais.

3. Folhas membranáceas, 3-5-partidas.

4. Face abaxial das folhas com tricomas simples e longos ( $>1 \mathrm{~mm}$ compr.) sobre as nervuras principais; lobos do cálice das flores pistiladas com tricomas simples e longos (ca. $1 \mathrm{~mm}$ compr.) . 1. A. digitata

4'. Face abaxial das folhas com tricomas estrelados ou simples e curtos $(<0.5 \mathrm{~mm}$ compr.) ao longo das nervuras principais; lobos do cálice das flores pistiladas glabros ou com tricomas simples e curtos $(<1 \mathrm{~mm}$ compr. $)$ 2. A. gracilis
3'. Folhas cartáceas, inteiras ou 2- ou 3-partidas. 5. Ramos jovens tomentosos

6. A. subcomosa

5'. Ramos jovens glabros, pilosos a pubescentes.

6. Estípulas deltoides; face abaxial das folhas glabras ou com indumento composto por tricomas simples

\section{A. klotzschii}

6'. Estípulas subuladas; face abaxial das folhas com indumento composto principalmente por tricomas estrelados 4. A. paulina

1. Astraea digitata (Müll.Arg.) O.L.M.Silva \& Cordeiro, Phytotaxa 317: 297. 2017.

Figuras 1A, B e 2.

Arbustos ou subartbustos perenes, até $1(-1,5) \mathrm{m}$ alt.; ramos jovens esparsamente pubescentes a glabrescentes, com tricomas simples e/ou estrelados. Folhas 3(-5)-partidas, lobo médio elíptico a largoelíptico, 5-8(-11) $\times$ 2,5-3(-4) $\mathrm{cm}$, lobos laterais elípticos a oblongos, $(2,5-) 4-7(-9,5) \times 1,5-2(-3) \mathrm{cm}$, membranáceas, base cordada a subcordada, face adaxial pubescente, indumento mais denso na base e ao longo das nervuras primárias, com tricomas simples e longos (>1 mm compr.), especialmente próximo da base, face abaxial densamente pubescente, indumento mais denso na base e ao longo das nervuras, com tricomas simples e curtos, exceto sobre as nervuras, onde são simples e longos ( $>1 \mathrm{~mm}$ compr.), nervuras actinódromas; pecíolos (1,5-)4-8,5(-10) cm compr.; estípulas subuladas, inteiras ou 3-lobadas. Tirsos 15-35 cm compr.; címulas estaminadas com 4 ou 5 flores cada. Flores pistiladas com pedicelo ca. $1(-5$, no fruto) $\mathrm{mm}$ compr.; lobos do cálice oblongos a estreito-obovados, $2-3,5$ (-8, no fruto) $\mathrm{mm}$ compr., pilosos na face externa, com tricomas simples e longos, ca. $1 \mathrm{~mm}$ compr.; ovário glabro. Cápsulas 4,5-5,5 × 4,5-5,5 mm, glabras; sementes 3-3,5 × 2,5-2,8 mm, carúncula $0,3-0,5 \times 0,8$ $1 \mathrm{~mm}$.

Restrita à Região Nordeste, ocorrendo em Alagoas, Bahia, Paraíba e Pernambuco. E8, E9, E/F9, F/G8, G8, G8/9 e H8/9: Mata Atlântica, em bordas de florestas úmidas. Encontrada com flores e/ou frutos o ano todo.

Material selecionado: Amélia Rodrigues, 23 mar. 1994 (fl., fr.), F. França 957 (HUEFS, SP); Camamu, 16 jun. 2003 (fl., fr.), G. Hatschbach 75396 (ALCB, MBM); Conceição do Almeida, 6 set. 1959 (fl., fr.), A.L. Costa s.n. (ALCB 2068); Cruz das Almas, 1950 (fl., fr.), G.C. Pinto s.n. (MBML 2473, SP 274822); Ilhéus, 8 fev. 2015 (fl., fr.), O.L.M. Silva 228 (SP); Salvador, 19 out. 2006 (fl., fr.), E.P. Queiroz 2173 (HRB); São Francisco do Conde, 22 jul. 1991 (fl., fr.), M.L. Guedes s.n. (ALCB 27934); Una, 18 set. 2004 (fl., fr.), W.W. Thomas 14137 (CEPEC, NY); Uruçuca, 16 mar. 1970 (fl., fr.), T.S. Santos 608 (CEPEC).

Astraea digitata é mais semelhante morfologicamente a A. gracilis pelas folhas membranáceas, sempre 3-5partidas. As duas espécies ocorrem em simpatria na 
região sul do estado e podem ser diferenciadas pelo indumento da face abaxial das folhas e da face externa dos lobos do cálice da flor pistilada: em $A$. digitata ambos são cobertos de tricomas simples e longos (ca. 1 mm compr.), enquanto em $A$. gracilis os tricomas simples são curtos ( $<0.5 \mathrm{~mm}$ compr.).

\section{Astraea gracilis (Müll.Arg.) O.L.M.Silva \& Cordeiro, Phytotaxa 317(4): 299. 2017.}

Figuras $1 \mathrm{C}-\mathrm{E}$ e 2.

Arbustos ou subarbustos perenes, até 1,5 m alt.; ramos jovens glabros ou pilosos a esparsamente pubescentes, com tricomas simples e/ou estrelados. Folhas 3(-5)-partidas, lobo médio estreito- a largoelíptico, levemente oblanceolado ou ovado, 3-8(-11,5) $\times(1-) 1,5-3(-4) \mathrm{cm}$, lobos laterais oblongos ou elípticos, $(2,5-) 3-7,5 \times 1-5 \mathrm{~cm}$, membranáceas, base arredondada, truncada ou levemente subcordada ou cuneada, face adaxial pubérula, puberulenta ou estrigosa, indumento mais denso na base e ao longo das nervuras primárias, com tricomas simples e curtos juntamente com estrelados ou estrelado-porrectos (especialmente na base e ao longo das nervuras primárias), face abaxial glabrescente a esparsamente puberulenta, indumento mais denso na base, com tricomas simples e curtos ou estrelados, nervuras actinódromas; pecíolos $(0,5-) 2,5-5(-12) \mathrm{cm}$ compr.; estípulas subuladas, inteiras ou 3-lobadas. Tirsos (7-)10-30 cm compr.; címulas estaminadas com 34(5) flores cada. Flores pistiladas com pedicelo 1-2(7, no fruto) mm compr.; lobos do cálice obovados a estreito-oblongos, 2-4(-15, no fruto) mm compr., esparsamente pubescentes na face externa, com tricomas simples ou longos; ovário glabro. Cápsulas 5-8 × 5-8 mm, glabras; sementes 3-5 × 2-4.5 mm, carúncula ca. $0.5 \times 1 \mathrm{~mm}$.

Espécie de ampla distribuição na América do Sul, registrada para a Guiana Francesa, Bolívia, Paraguai, Argentina e Brasil. No Brasil, ocorre em Alagoas, Bahia, Ceará, Espírito Santo, Goiás, Mato Grosso, Mato Grosso do Sul, Minas Gerais, Pernambuco, Rio de Janeiro e São Paulo. D6, E3, E8, G5, G8, G8/9 e H8: Caatinga, Cerrado e Mata Atlântica, em bordas de matas úmidas, florestas estacionais semidecíduas e decíduas ou em afloramentos rochosos. Encontrada com flores e/ou frutos o ano todo.

Material selecionado: Anguera, 31 out. 2006 (fl., fr.), J.S. Novais 24 (HUEFS, SP); Arataca, 6 fev. 2015 (fl., fr.), O.L.M. Silva 225 (SP); Barreiras, 15 maio 2009 (fl., fr.), B.T.C. Santos 476 (HUEFS); Ilhéus, 26 nov. 2015 (fl., fr.), I. Cordeiro 3554 (SP). Itabuna, 25 mar. 1965 (fl., fr.), R.P. Belém 534 (CEPEC, NY); Itacaré, 4 fev. 2015 (fl., fr.), O.L.M. Silva 222 (SP); Licínio de Almeida, 22 jan. 2013 (fl., fr.), F. Hurbath 451 (ALCB, SP); São Gabriel, 2 abr. 2009 (fl., fr.), R.F. Machado 104 (HUEFS, SP).

Astraea gracilis é morfologicamente próxima de $A$. digitata e a distinção entre as duas se dá pelo indumento da face abaxial das folhas e da face externa dos lobos do cálice da flor pistilada (ver comentário em A. digitata). Esta espécie foi tratada como A. lobata por Hurbath et al. (2016), no entanto $A$. gracilis se distingue de $A$. lobata pelos frutos lustrosos (vs. opacos em $A$. lobata). Astraea lobata, que é uma espécie tipicamente ruderal, não ocorre na Bahia, enquanto $A$. gracilis ocorre em matas úmidas, decíduas ou semidecíduas e em afloramentos rochosos.

3. Astraea klotzschii Didr., Vidensk. Meddel. Naturhist. Foren. Kjøbenhavn 1857: 137. 1857.

Figuras $1 F$, G e 2.

Arbustos perenes, até $2(-3,5) \mathrm{m}$ alt.; ramos jovens glabros ou pilosos, com tricomas simples e/ou estrelados. Folhas inteiras, ou às vezes (2)3-partidas, as inteiras elípticas, oblongas ou ovadas, $(1,5-) 3-9(-$ 11) $\times(0,7-) 1-4,5(-6)$; as partidas com lobo médio elíptico, oblongo ou levemente ovado, $(4,5-) 5-8(-9) \times$ (1-)1,5-3,5(-4) cm, lobos laterais oblongos ou elípticos, 3-4 × 0,5-1,5 cm, cartáceas, base cuneada, obtusa, arredondada ou subcordada, face adaxial glabra ou pilosa na base e/ou ao longo das nervuras primárias, com tricomas simples e curtos $(<0.5 \mathrm{~mm}$ compr.), face abaxial glabra ou pilosa a pubescente, indumento mais denso na base, com tricomas simples e curtos, geralmente na base ou ao longo das nervuras principais, camptódromas (inteiras) ou actinódromas (partidas); pecíolos $(0,5-) 1-4,5(-5,5)$ cm compr.; estípulas deltoides, inteiras. Tirsos (10-)15-25(-30) cm compr.; címulas estaminadas com 3-5(6) flores cada. Flores pistiladas com pedicelo $0,5-2(-5$, no fruto) $\mathrm{mm}$ compr.; lobos do cálice oblongos, elípticos ou levemente obovados, 3-5(-9, no fruto) mm compr., glabros, pilosos ou pubescentes na face externa, com tricomas simples e curtos; ovário glabro ou raramente com poucos tricomas simples e curtos na porção apical. Cápsulas 5,5-6,5 × 5,5-6,5 mm, glabras ou raramente com poucos tricomas simples e curtos na porção apical; sementes $4-5 \times 2,5-4 \mathrm{~mm}$, carúncula $1-1,5 \times 0.5-1$ $\mathrm{mm}$.

Espécie endêmica do Brasil, com ampla distribuição ao longo da costa Atlântica, em Alagoas, Bahia, Ceará, Espírito Santo, Paraíba, Pernambuco, Rio de Janeiro e Sergipe. D10, E5/6, E6, E9, E10, E/F6, F8/9, G8/9, H8/9, I8, I8/9, J8 e K8: Cerrado e Mata Atlântica, em restingas ou, mais raramente, em campos rupestres. Encontrada com flores e/ou frutos o ano todo.

Material selecionado: Alagoinhas, 25 jan. 2001 (fl., fr.), L.E. Figueiroa 46 (HUEFS); Alcobaça, 17 set. 1978 (fl., fr.), S.A. Mori 10579 (CEPEC, DAV, MO, NY, SPF); Barrolândia, 17 jul. 2006 (fl., fr.), J. Paula-Souza 6053 (ESA, SP); Belmonte, 9 fev. 2012 (fl., fr.), M.L. Guedes 19345 (ALCB); Cairu, 3 jan. 2007 (fl., fr.), A.M.A. Amorim 6768 (CEPEC, NY, SP); Camaçari, 15 fev. 2004 (fl., fr.), M.I.B. Silva 3 (ALCB); Canavieiras, 21 fev. 1970 (fl., fr.), J.A. Jesus 563 (CEPEC, IPA); Conceição da Barra, 10 jun. 2003 (fl., fr.), G. Hatschbach 75110 (ALCB, HUFU, MBM); Conceição do Jacuípe, 7 mar. 2003 (fl., fr.), M.V. Moraes 573 (NY); Conde, 4 set. 2003 (fl., fr.), N.G. Jesus 1828 (HRB); Entre Rios, 28 jan. 2011 (fl., fr.), F.S. Gomes 852 (ALCB); Esplanada, 9 maio 2000 (fl., fr.), N.G. Jesus 934 (ALCB, CEPEC, HRB, SPF, UESC); Eunápolis, 23 

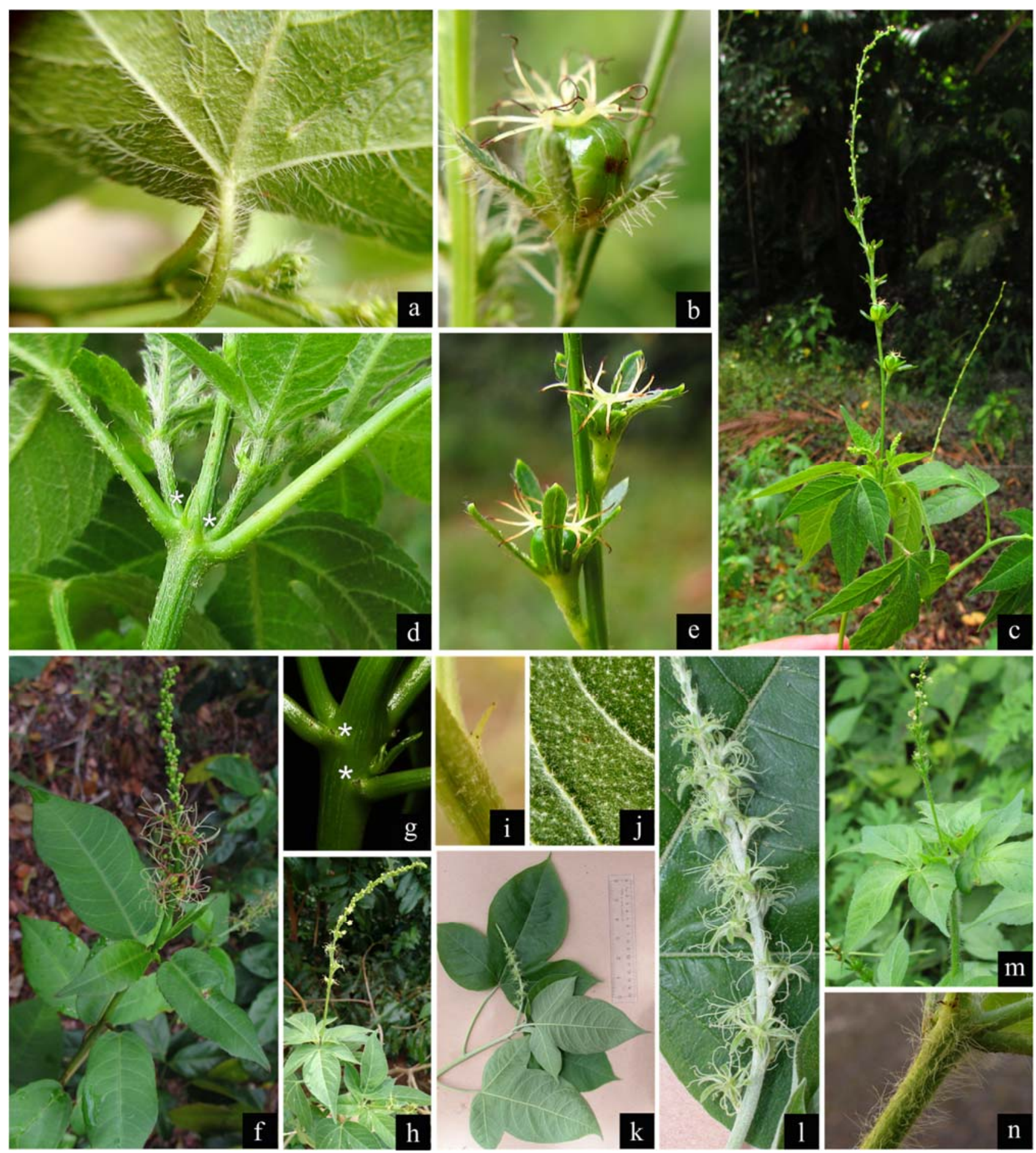

Figura 1. A, B. Astraea digitata: A- detalhe do pecíolo e face abaxial da lâmina foliar evidenciado os tricomas simples e longos sobre as nervuras; B- flor pistilada. C-E. A. gracilis: C- ramo com inflorescência; D- detalhe da inserção das folhas, evidenciando as estípulas (*), pecíolos e parte da face abaxial das folhas; E- flores pistiladas. F, G. A. klotzschii: F- ramo com inflorescência; G- detalhe da inserção da folha, evidenciando estípulas (*). H-J. A. paulina: H- ramo com inflorescência; I- estípulas; J- detalhe do indumento da face abaxial da folha. K, L. A. praetervisa: K- ramo com inflorescência; L- inflorescência evidenciando as flores pistiladas. M, N. A. surinamensis: M- ramos com inflorescência; N- detalhe do indumento de um ramo jovem. (Fotos: A-J e M-N: O.L.M. Silva; K-L: P.E. Berry.)

set. 1968 (fl., fr.), J. Almeida 83 (CEPEC, DAV, R, RB); Ilhéus, 25 out. 2010 (fl., fr.), I. Cordeiro 3235 (SP); Itacaré, 24 nov. 2015 (fl., fr.), I. Cordeiro 3550 (SP); Jaguaripe, 23 nov. 2016 (fl., fr.), G. Costa 2142 (SP); Lauro de Freitas, 9 jul. 2003 (fl., fr.), M.L. Guedes 10818 (ALCB); Lençóis, 28 jan. 1997 (fl., fr.), S. Atkins in PCD4594 (ALCB); Maraú, 2 out. 2013 (fl., fr.), M.L. Guedes 21145 (ALCB, SP); Mata de São João, 25 out. 2014 (fl., fr.), O.L.M. Silva 195 (SP);
Mucugê, 7 jan. 2012 (fl., fr.), M. Alves 47 (ALCB); Mucuri, 4 out. 2000 (fl., fr.), L.A. Mattos-Silva 4153 (ALCB, CEPEC, HRB, HUEFS, NY, UESC); Nova Viçosa, 5 out. 2000 (fl., fr.), L.A. MattosSilva 4187 (ALCB, CEPEC, UESC); Palmeiras, 31 ago. 1996 (fl., fr.), A.A. Conceição 180 (SP, SPF); Porto Seguro, 29 fev. 2014 (fl., fr.), M.L. Guedes 23230 (ALCB, UB); Prado, 21 out. 1993 (fl., fr.), W.W. Thomas 10070 (CEPEC, SP); Salvador, 15 jul. 2002 (fl., fr.), 


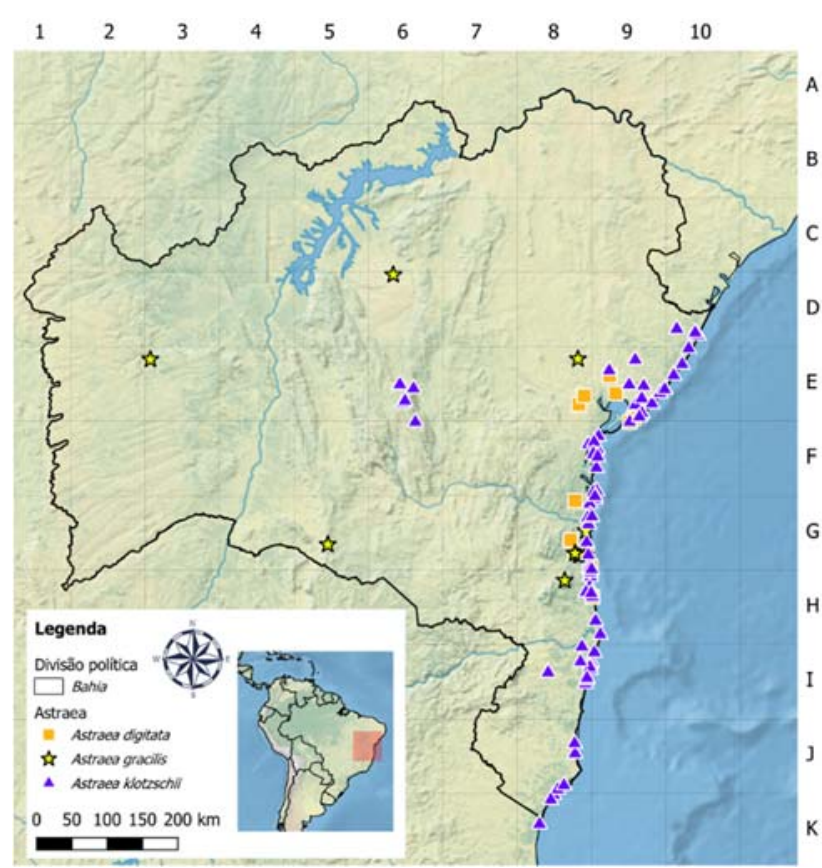

Figura 2. Mapa de distribuição geográfica de Astraea digitata, A. gracilis e A. klotzschii no estado da Bahia.

M.L. Guedes 9615 (ALCB); Santa Cruz Cabrália, 19 fev. 2004 (fl., fr.), M.L. Guedes 11105 (ALCB); Santo Antônio, $27 \mathrm{dez}$. 2005 (fl., fr.), T.S. Nunes 1414 (NY, UESC); São Sebastião do Passé, 20 out. 1998 (fl., fr.), A.F.S. Nascimento 190 (ALCB, CEPEC); Simões Filho, 18 out. 1986 (fl., fr.), M.L. Guedes 1192 (ALCB); Una, 22 out. 2011 (fl., fr.), E.N. Matos 701 (SP); Valença, 14 jan. 1997 (fl., fr.), M.M. Arbo 7173 (CEPEC, DAV, NY, SP).

Astraea klotzschii é um exemplo de distribuição disjunta entre restingas e campos rupestres, mas a grande maioria de suas coleções são provenientes de restingas. Geralmente apresenta folhas glabras, exceto pelas margens ciliadas, porém em raros casos foram observados indivíduos com indumento de tricomas simples mais desenvolvido na face abaxial das folhas, bem como na face externa dos lobos do cálice. Como em muitas espécies do gênero, A. klotzschii possui heterofilia, lâminas partidas ou inteiras, com predomínio dessas últimas.

Pode ser distinguida de Astraea praetervisa, a outra espécie das restingas baianas, pelos ramos jovens glabros ou pilosos, estípulas deltoides e folhas geralmente glabras (vs. ramos jovens tomentosos, estípulas glanduliformes e folhas com face abaxial tomentosa em A. praetervisa). Exemplares dos campos rupestres podem ser confundidos com $A$. subcomosa pelo predomínio de folhas inteiras; com A. macroura (Colla) P.L.R.Moraes, De Smedt \& Guglielmone por também ocorrer em restingas (porém restrita aos estados do Espírito Santo e Rio de Janeiro) e predominância de folhas inteiras; e com A. paulina pelo indumento não muito desenvolvido, porém as estípulas deltoides de $A$. klotzschii são únicas no gênero e permitem distingui-la de $A$. subcomosa, $A$. macroura e A. paulina, que possuem estípulas subuladas geralmente bem desenvolvidas.
4. Astraea paulina Didr., Vidensk. Meddel. Naturhist. Foren. Kjøbenhavn 1857: 138. 1857.

Figuras $1 \mathrm{H}-\mathrm{J}$ e 3.

Arbustos perenes, até $2 \mathrm{~m}$ alt.; ramos jovens pilosos a pubescentes, geralmente com tricomas estrelados, mas às vezes também com esparsos tricomas simples curtos ou estrelado-porrectos, com o raio central não muito maior que os laterais. Folhas inteiras ou (2)3(-5)partidas, as inteiras ovadas, $(2-) 2,5-8(-13) \times 1-4(-6)$ $\mathrm{cm}$, as partidas com lobo médio elíptico, oblongo ou ovado, $(2-) 3-8(-15) \times(0,5-) 2-4,5(-5,5) \mathrm{cm}$, lobos laterais elípticos ou oblongos, $(0,5-) 2-6(-10) \times$ $(0,3-) 0,5-4,5) \mathrm{cm}$, cartáceas, base arredondada, cordada, subcordada, truncada ou obtusa, face adaxial pilosa, pubescente ou com tricomas esparsos, indumento mais denso na base e ao longo das nervuras primárias, com tricomas simples e curtos $(<0,5 \mathrm{~mm}$ compr.) ou estrelados, face abaxial esparsa a densamente pubescente ou tomentosa, indumento mais denso na base e ao longo das nervuras, principalmente com tricomas estrelados, às vezes estrelado-porrectos ou simples curtos, nervuras camptódromas (inteiras) ou actinódromas (partidas); pecíolos $(0,2-) 0,5-7(-10) \mathrm{cm}$ compr.; estípulas subuladas, inteiras ou 3(-5)-lobadas. Tirsos 8-20(-35) cm compr.; címulas estaminadas com $2-5$ flores cada. Flores pistiladas com pedicelo $0.5-2(-$ 6, no fruto) mm compr.; lobos do cálice oblongos, elípticos ou levemente ovados ou obovados, $2-4(-6$, no fruto) mm compr., pilosos, pubescentes ou tomentosos na face externa, com tricomas estrelados, estreladoporrectos ou simples e curtos; ovário glabro, piloso ou pubescente, com tricomas estrelados ou simples e curtos. Cápsulas 5-5,5(-6) × 5-5,5(-6) mm, glabras a pilosas, com tricomas estrelados ou simples e curtos; sementes 4-5 × 2.5-3 mm, carúncula ca. 0,5 × $1.5 \mathrm{~mm}$.

Espécie também encontrada na Bolívia e Paraguai. No Brasil, ocorre em Alagoas, Bahia, Ceará, Distrito Federal, Goiás, Maranhão, Mato Grosso, Minas Gerais, Pernambuco, São Paulo, Tocantins e Pará. C6, C7, D6, D6/7, D7, E6, E6/7, E7, F5, F5/6, F6, G6/7 e H7: Caatinga, Cerrado e Mata Atlântica, em bordas de matas semidecíduas, matas de galeria e campos rupestres. Encontrada com flores e/ou frutos o ano todo.

Material selecionado: Abaíra, 10 mar. 1992 (fl. fr), B.L. Stannard H51838 (HUEFS, K, NY, SP, SPF); Água Quente, 16 dez. 1988 (fl., fr.), R.M. Harley 27194 (CEPEC, K, NY, SP, SPF); Andaraí, 24 jan. 2010 (fl., fr.), R.B. Santos 45 (HUEFS); Bonito, 11 nov. 1998 (fl., fr.), D.S. Carneiro 62 (CEPEC, HUEFS, SP); Itaberaba, 21 set. 2005 (fl., fr.), D. Cardoso 801 (HUEFS, NY, SP); Itaeté, 21 fev. 2004 (fl., fr.), R. Funch 148 (HUEFS); Jacobina, 24 nov. 2001 (fl., fr.), D.M. Loureiro 465 (ALCB, CEPEC); Lençóis, 25 ago. 2014 (fl., fr.), O.L.M. Silva 184 (SP); Miguel Calmon, 5 abr. 2001 (fl., fr.), H.P. Bautista 3029 (CEPEC, HRB, HUEFS); Mirangaba, 1 set. 1981 (fl., fr.), L.M.C. Gonçalves 167 (CEPEC, HRB, MG, RB); Morro do Chapéu, 27 set. 1978 (fl., fr.), G. Martinelli 5311 (RB); Mucugê, 18 nov. 2005 (fl., fr.), D.S. CarneiroTorres 599 (HRB, HUEFS, SP); Nova Redenção, 2 mar. 2003 (fl., fr.), L.R. Senna 157 (HUEFS); Palmeiras, 25 jul. 2014 (fl., fr.), F. Hurbath 666 (ALCB); Piatã, 19 jan. 2006 (fl., fr.), A.A. Conceição 


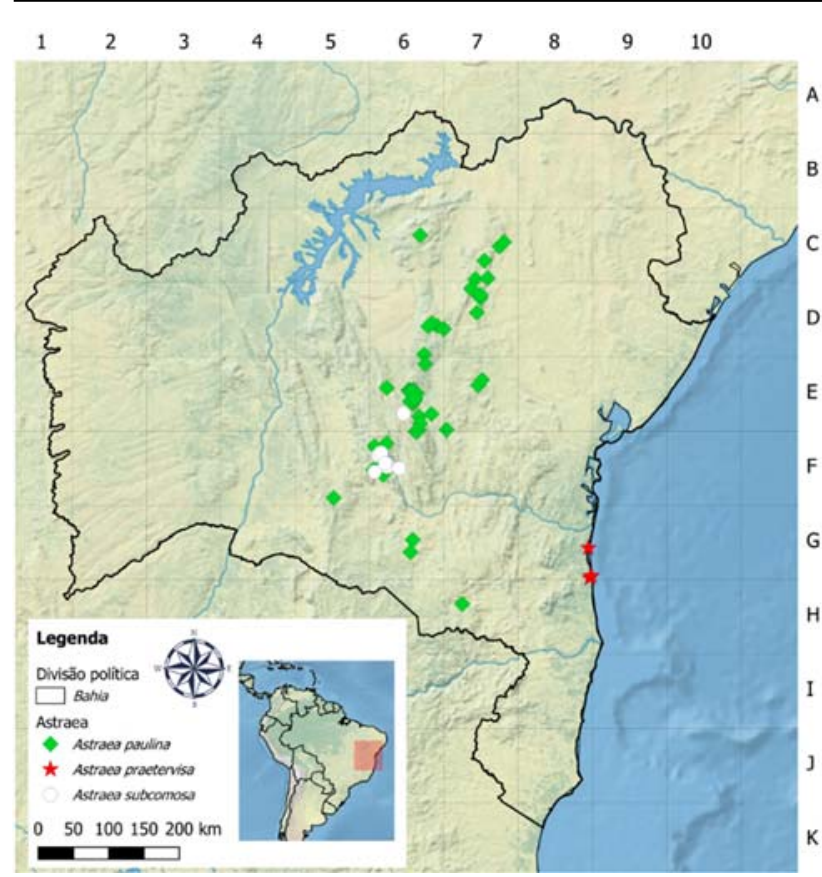

Figura 3. Mapa de distribuição geográfica de Astraea paulina, A. praetervisa e A. subcomosa no estado da Bahia.

1679 (HUEFS, SP); Pindobaçu, 10 abr. 2001 (fl., fr.), T. Ribeiro 209 (ALCB, CEPEC, HRB, HUEFS, UESC); Ribeirão do Largo, 14 ago. 2001 (fl., fr.), A.M.V. Carvalho 6905 (ALCB, CEPEC, HRB, HUEFS, NY, SP, UESC); Rio de Contas, 16 nov. 2012 (fl., fr.), R.F. Monteiro 642 (RB, SP); Rio do Pires, 24 jul. 1993 (fl., fr.), W. Ganev 1943 (HUEFS, K, NY, SP); Ruy Barbosa, 18 dez. 2004 (fl., fr.), L.P. Queiroz 9926 (HUEFS, SP); Seabra, 4 dez. 1980 (fl., fr.), N.L. Menezes in CFCR 384 (SPF, WIS); Senhor do Bonfim, 28 out. 2005 (fl., fr.), S.F. Conceição 302 (CEPEC, ESA, HUEFS); Umburanas, 9 abr. 1999 (fl., fr.), L.P. Queiroz 5179 (HUEFS, SP); Utinga, 19 nov. 1986 (fl., fr.), G.L. Webster 25769 (DAV).

Astraea paulina exibe grande plasticidade quanto à densidade do indumento e o formato do limbo foliar, que pode ser exclusivamente inteiro ou partido ou dos dois tipos em um mesmo indivíduo. Na Bahia, A. paulina ocorre principalmente ao longo da Cadeia do Espinhaço, e se distingue de $A$. subcomosa, outra espécie encontrada nessa região, pelos ramos jovens pilosos a pubescentes (vs. tomentosos em A. subcomosa). Embora incomum em campos rupestres da Bahia Central, $A$. klotzschii se distingue de A. paulina pelas suas estípulas deltoides (vs. subuladas em A. paulina). Por fim, também pode ser confundida com $A$. gracilis, mas esta última possui folhas membranáceas, enquanto em $A$. paulina, elas são cartáceas.

\section{Astraea praetervisa (Müll.Arg.) P.E.Berry, Amer. J.} Bot. 92: 1532. 2005.

Figuras 1K, L e 3.

Arbustos perenes, até $2 \mathrm{~m}$ alt.; ramos jovens tomentosos, com tricomas estrelados. Folhas inteiras ou 2- ou 3-partidas, as inteiras elípticas, oblongas ou largoovadas, $6,5-10 \times 2,5-5 \mathrm{~cm}$, as partidas com lobo médio elíptico, $(6,5-) 9-15(-18) \times(2,5-) 4-6,5(-7,5) \mathrm{cm}$, lobos laterais elípticos, (4-)5-12 $\times(2-) 4-4,5(-6) \mathrm{cm}$, cartáceas, base truncada a cordada, face adaxial pilosa, exceto pelas nervuras primárias densamente pubescentes, com tricomas estrelados e estreladoporrectos, face abaxial tomentosa, indumento mais denso na base e ao longo das nervuras, com tricomas estrelados e estrelado-porrectos, nervuras actinódromas; pecíolos (2-)4-10(-13) cm compr.; estípulas glanduliformes, inteiras. Tirsos $20-35(-55)$ cm compr.; címulas estaminadas com 4 ou 5(6) flores cada. Flores pistiladas com pedicelo $1-2(-3$, no fruto) $\mathrm{mm}$ compr.; lobos do cálice estreito-oblongos (linear no fruto), 5-7(12, no fruto) $\mathrm{mm}$ compr., tomentosos na face externa, com tricomas estrelados; ovário piloso, com tricomas estrelados. Cápsulas 8-10 × 8-10 mm, glabras a glabrescentes, com tricomas estrelados; sementes 5-5,5 $\times$ 3-4 mm, carúncula ca. $0,5 \times 1,5 \mathrm{~mm}$.

Encontrada apenas na região de Ilhéus. G8/9: Mata Atlântica, em restingas. Os raros registros desta espécie foram realizados em outubro e janeiro, podendo a floração desta espécie estar associada com a temporada úmida.

Material examinado: Ilhéus, 19 fev. 2004 (fl.), S.C. Sant'Ana 1123 (CEPEC, MICH, NY, RB); 12 fev. 1994 (fl., fr.), J.R. Pirani 2938 (K, MO, SP, SPF, WIS); 22 out. 1983 (fl., fr.), A.M.V. Carvalho 2005 (ALCB, G, HUEFS, MBM); 3 fev. 1993 (fl., fr.), W.W. Thomas 9709 (CEPEC, DAV, MBM, NY, R, SP).

Astraea praetervisa pode ser prontamente identificada entre as espécies que ocorrem em restingas por suas estípulas glanduliformes e pelo indumento tomentoso, com tricomas estrelados.

\section{Astraea subcomosa (Müll.Arg.) Caruzo, Phytotaxa} 159(2): 127. 2014.

Figura 3 (veja também fig. 29 em Silva \& Cordeiro 2020).

Arbustos perenes, até $2 \mathrm{~m}$ alt.; ramos jovens tomentosos, com tricomas estrelados e estreladoporrectos, com o raio central não maior que os laterais. Folhas na maioria inteiras, ocasionalmente com algumas 2- ou 3-partidas, as inteiras ovadas, oblongas ou elípticas, $(3-) 5-10(-14,5) \times 2-4,5 \mathrm{~cm}$, as partidas com lobo médio elíptico, 4,5-11,5 × 2,5-5 cm, lobos laterais elípticos a oblongos, 5,5-6 × 1-2 cm, cartáceas, base obtusa a arredondada, nas partidas às vezes cordada, face adaxial pilosa, exceto pelas nervuras primárias densamente pubescentes, com tricomas estrelados e estrelado-porrectos e poucos simples e curtos $(<0,5 \mathrm{~mm})$, face abaxial tomentosa, indumento mais denso na base e ao longo das nervuras, com tricomas estrelados ou estrelado-porrectos, nervuras actinódromas; pecíolos $0,5-2(-5) \mathrm{cm}$ compr.; estípulas subuladas, inteiras. Tirsos $20-45 \mathrm{~cm}$ compr.; címulas estaminadas com 4 ou 5(6) flores cada. Flores pistiladas com pedicelo $1-2(-9$ no fruto) $\mathrm{mm}$ compr.; lobos do cálice elípticos a obovados, $3-3,5(-8$, no fruto) mm compr., pubescentes na face externa, com tricomas estrelados, estrelado-porrectos ou simples e curtos; ovário glabro a pubescente, mais denso na porção apical, com tricomas simples curtos e estrelados. Cápsulas 6$6,5(-8) \times 6-6,5(-8) \mathrm{mm}$, esparsamente pubescentes, indumento mais denso na porção apical, com tricomas 
simples curtos e estrelados; sementes $4,5-5(5,5) \times 3-$ $3,5(-4) \mathrm{mm}$, carúncula ca. $0,5(-1) \times 1(-2) \mathrm{mm}$.

Encontrada apenas ao longo da Cadeia do Espinhaço, nos estados de Minas Gerais e Bahia. E6, F6: em matas de galeria, borda de florestas sazonais ou campos rupestres. Encontrada com flores e/ou frutos de outubro a maio.

Material selecionado: Abaíra, 25 maio 1992 (fl., fr.), W. Ganev 375 (HUEFS, NY, SP, SPF); Mucugê, 29 jun. 2002 (fl., fr.), A.A. Conceição 1069 (SP, SPF); Rio de Contas, 1 fev. 2003 (fl., fr.), L.R. Lima 261 (SP, SPF).

Dentre as espécies da Bahia, Astraea subcomosa pode ser confundida com A. paulina pelas folhas inteiras e partidas. Uma comparação entre as duas é apresentada em A. paulina. Outra espécie semelhante a $A$. subcomosa é A. comosa (Müll.Arg.) B.W. van Ee, porém esta última possui folhas predominantemente 3partidas, e é endêmica de Minas Gerais.

7. Astraea surinamensis (Miq.) O.L.M.Silva \& Cordeiro, Phytotaxa 404: 133. 2019.

Figuras 1M, N e 4.

Ervas anuais ou bianuais, até $1,5 \mathrm{~m}$ alt.; ramos jovens esparsamente a densamente pubescentes, com tricomas estrelado-porrectos com o raio central muito maior que os laterais (até 3-4 mm compr.), às vezes também com tricomas simples e longos $(>1.5 \mathrm{~mm}$ compr.). Folhas 3(-5)-partidas, lobo médio elíptico a largo-elíptico, $(1,5-) 2-9(-10) \times(0,7-) 1-4,5(-5) \mathrm{cm}$, lobos laterais elípticos ou oblongos, $(1-) 1,5-5(-6,5) \times$ $0,5-3(-3,5) \mathrm{cm}$, membranáceas, base arredondada, cordada, subcordada, truncada, obtusa ou cuneada, face adaxial glabrescente a pilosa, indumento mais denso perto da base, junto das nervuras primárias, principalmente com tricomas simples e longos ( $>1 \mathrm{~mm}$ compr.), mas também com esparsos estrelados ou

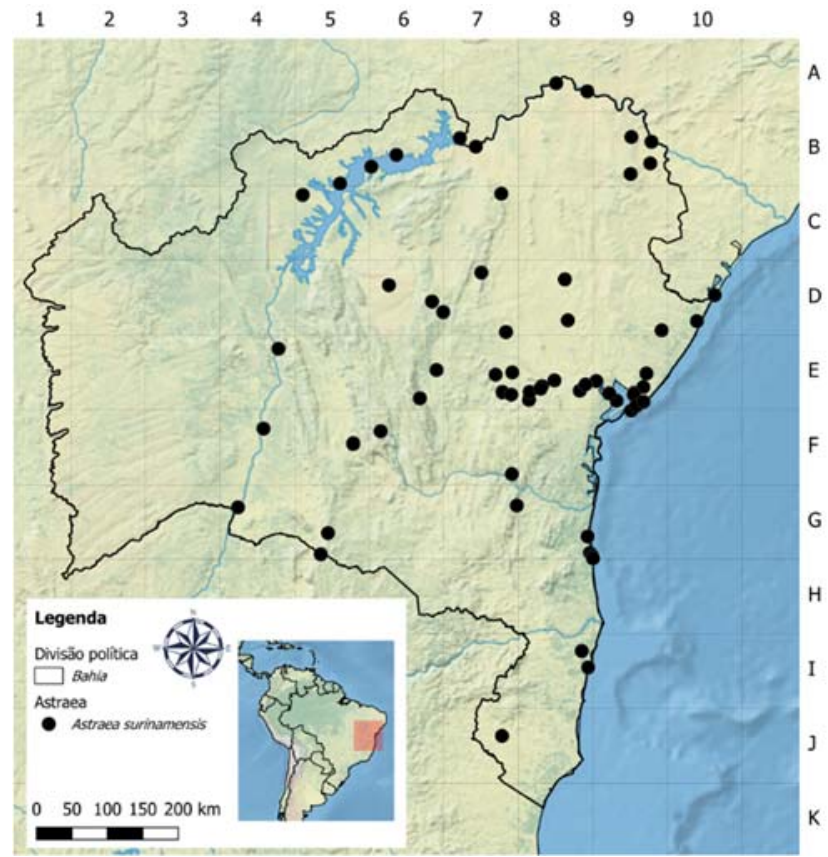

Figura 4. Mapa de distribuição geográfica de Astraea surinamensis no estado da Bahia. estrelado-porrectos com o raio central muito maior que os laterais, especialmente na base ou ao longo das nervuras primárias, face abaxial pilosa, indumento mais denso na base, principalmente com tricomas simples longos ou curtos, mas também com tricomas estrelados ou estrelado-porrectos com raio central superando $1 \mathrm{~mm}$ compr., especialmente perto da base ou ao longo das nervuras, actinódromas; pecíolos $(0,5) 1-5(-7) \mathrm{cm}$ compr.; estípulas subuladas, inteiras ou 3(-5)-lobadas. Tirsos 8-15(-20) cm compr.; címulas estaminadas com 1-3 flores cada. Flores pistiladas com pedicelo 2-3(5 , no fruto) $\mathrm{mm}$ compr.; lobos do cálice elípticos ou oblongos, $2-3(-7$, no fruto) $\mathrm{mm}$ compr., pilosos na face externa, com tricomas simples e longos; ovário pubescente a densamente pubescente, com tricomas simples e longos, raramente com tricomas estreladoporrectos com raio central muito maior que os laterais. Cápsulas 4-5(-6) × 4-5(-6) $\mathrm{mm}$, pilosas a esparsamente pubescentes, com tricomas simples e longos, raramente com tricomas estrelado-porrectos com raio central muito maior que os laterais; sementes $(3,5-) 4-5 \times 2,5-3 \mathrm{~mm}$, carúncula ca. $0,5 \times 1,5 \mathrm{~mm}$.

Espécie ruderal de ampla distribuição na América Tropical. No Brasil, só não é encontrada na Região Sul. A8, B5, B6, B7, B9, C5, C7, D6, D7, D8, D9, D10, E4, E6, E7, E8, E9, F4, F5, F6, F7, G4, G5, G7/8, G8/9, I8 e J7: Caatinga, Cerrado e Mata Atlântica, em ambientes alterados e antrópicos. Encontrada com flores e/ou frutos o ano todo.

Material selecionado: Abaíra, 29 abr. 2006 (fl., fr.), M.L. Guedes 12243 (ALCB); Abaré, 1 nov. 2009 (fl., fr.), E. Melo 7055 (HUEFS, SP); Andaraí, 19 out. 1997 (fl., fr.), M. Alves EBNN 1144 (UFP); Baixa Grande, 21 jul. 2006 (fl., fr.), J. Paula-Souza 6346 (ESA); Bom Jesus da Lapa, 29 abr. 2003 (fl., fr.), N. Roque 709 (ALCB, CEPEC); Cachoeira, jun. 1980 (fl., fr.), Grupo Pedra do Cavalo 115 (ALCB, CEPEC, HRB, HUEFS, IPA, NY, RB); Camaçari, 18 out. 1996 (fl., fr.), D.S. Carneiro 7 (HUEFS, UB); Carinhanha, 17 maio 2000 (fl., fr.), G. Hatschbach 71273 (MBM); Casa Nova, 9 fev. 2004 (fl., fr.), L.P. Queiroz 9121 (HUEFS, SP); Castro Alves, 9 mar. 2008 (fl., fr.), S.F. Conceição 577 (HUEFS, SP); Conde, 20 jun. 2003 (fl., fr.), G. Hatschbach 75573 (CEPEC, G, HRCB, MBM); Cruz das Almas, 17 nov. 2000 (fl., fr.), A.A.C. Rodrigues 2 (ALCB); Entre Rios, 7 abr. 2009 (fl., fr.), A.V. Popovkin 547 (HUEFS, SP); Glória, 1 jul. 1995 (fl., fr.), F.P. Bandeira 167 (HUEFS, MBM, UB); Iaçu, 22 fev. 1997 (fl., fr.), E. Melo 2063 (HUEFS, MBM, SP); Ibotirama, 11 out. 2007 (fl., fr.), J. PaulaSouza 9349 (SP, SPF); Ilhéus, 9 fev. 2015 (fl., fr.), O.L.M. Silva 230 (SP); Itaberaba, 5 jun. 2005 (fl., fr.), E. Melo 3906 (CEPEC, HUEFS, SP); Itagi, 24 ago. 2011 (fl., fr.), R.S. Souza 6 (UFP); Itaparica, 9 mar. 1989 (fl., fr.), E. Queiroz s.n. (HRB 30896, RB 482298); Itatim, 21 ago. 2014 (fl., fr.), O.L.M. Silva 147 (SP); Jacobina, 20 abr. 2009 (fl., fr.), M.L. Guedes 14704 (ALCB); Jaguarari, 13 abr. 2006 (fl., fr.), R.F. Souza-Silva 156 (CEPEC, HUEFS); Jandaíra, 2 abr. 1988 (fl., fr.), M.C. Ferreira 165 (HRB, RB); Jequié, 14 jul. 1979 (fl., fr.), S.A. Mori 12197 (CEPEC, NY, RB, US); João Dourado, 10 abr. 2013 (fl., fr.), M.L. Guedes 20655 (ALCB); Juazeiro, 5 mar. 2013 (fl., fr.), M.L. Guedes 20913 (ALCB); Lauro de Freitas, 6 mar. 1989 (fl., fr.), R. Soeiro 38/89 (HRB); Licínio de Almeida, 21 fev. 2014 (fl., fr.), F. Hurbath 620 
(ALCB); Mata de São João, 12 jan. 2004 (fl., fr.), L.P. Vieira 53 (ALCB); Medeiros-Neto, 25 jan. 2008 (fl., fr.), J.A. Lombardi 7145 (HRCB, SP); Milagres, 4 nov. 2010 (fl., fr.), E. Barbosa 2745 (MBM); Morro do Chapéu, 20 abr. 2013 (fl., fr.), F. Hurbath 531 (ALCB); Paramirim, 30 jan. 2008 (fl., fr.), J.L. Ferreira 279 (HUEFS, SP); Paulo Afonso, 10 jul. 2005 (fl., fr.), D.V. Braga s.n. (IPA 73896); Pilão Arcado, 19 mar. 2006 (fl., fr.), D.S. CarneiroTorres 626 (FUEL, HRCB, HUEFS); Porto Seguro, 29 abr. 2016 (fl., fr.), M.L. Guedes 24737 (ALCB); Riachão do Jacuípe, 28 dez. 2011 (fl., fr.), F. França 6178 (HUEFS); Salvador, 14 fev. 1998 (fl., fr.), J. Costa 138 (ALCB, HRB, HUEFS, UESC, IBGE); Santa Brígida, 23 ago. 2005 (fl., fr.), D.S. Carneiro-Torres 493 (HUEFS); Santa Cruz Cabrália, 23 jul. 1984 (fl., fr.), G.L. Webster 25028 (CEPEC); Santa Luz, 7 jul. 1976 (fl., fr.), A. Rocha s.n. (IAC 23271); Santa Teresinha, 2 jun. 1993 (fl., fr.), L.P. Queiroz 3187 (CEN, CEPEC, HUEFS, K, MBM); Sapeaçu, 13 out. 2010 (fl., fr.), M.L. Maia 1 (HUEFS); Sento Sé, 27 fev. 2008 (fl., fr.), C. Correia 416 (HUEFS); Simões Filho, 20 maio 2002 (fl., fr.), L.J. Alves 430 (ALCB, CEPEC); Urandi, 4 ago. 2009 (fl., fr.), M.L. Guedes 15764 (ALCB, HUEFS, MBM); Wagner, 11 mar. 2016 (fl., fr.), M.L. Guedes 24296 (UFRN).

Astraea surinamensis é a espécie ruderal do gênero com mais ampla distribuição no território brasileiro, especialmente na Região Nordeste. Se distingue de todas as demais por apresentar, nos ramos jovens e pecíolo, tricomas estrelado-porrectos, com raio central muito maior que os laterais, que chega a 3-4 mm compr. A olho nu, tais tricomas podem parecer simples e longos, e por serem rígidos ao toque, não é incomum ser popularmente confundida com cansansão (Cnidoscolus spp., Euphorbiaceae). É notável que esta espécie tenha sido originalmente descrita em Cnidoscolus (como C. surinamensis Miq.).

\section{AGRADECIMENTOS}

Agradecemos aos curadores dos herbários citados na lista de exsicatas, pelo acesso às coleções e empréstimos quando possível; ao Paul Berry, por ceder fotografias de Astraea praetervisa; à FAPESP, pelas bolsas de estudos ao primeiro autor (2013/26501-6 e 2017/06171-2); e ao $\mathrm{CNPq}$, pela bolsa de produtividade ao último autor
(309917/2015-8) e pelo financiamento do Projeto Flora da Bahia (483909/2012).

\section{REFERÊNCIAS}

BFG - The Brazil Flora Group. 2015. Growing knowledge: an overview of Seed Plant diversity in Brazil. Rodriguésia 66: 1-29.

Carneiro-Torres, D. S., Cordeiro, I., França, F. 2014. Euphorbiaceae. In: F. França \& E. Melo (Orgs), Flora de Inselbergues no Semiárido da Bahia 1. Universidade Estadual de Feira de Santana, Feira de Santana, p. 174-186.

Carneiro-Torres, D.S., Silva, O.L.M. \& Cordeiro, I. 2017. Flora da Bahia: Euphorbia (Euphorbiaceae). Sitientibus série Ciências Biológicas 17: 10.13102/scb2676

Cordeiro, I. 1995. Euphorbiaceae. In: B. Stannard (Org), Flora of the Pico das Almas, Bahia, Brazil. Royal Botanic Gardens, Kew, p. 300-317.

Flora do Brasil 2020, em construção. Jardim Botânico do Rio de Janeiro. Disponível em: < http://floradobrasil.jbrj.gov.br/ >. Acesso em: 07 abr. 2020.

Hurbath, F., Carneiro-Torres, D.S. \& Roque, N. 2016. Euphorbiaceae na Serra Geral de Licínio de Almeida, Bahia, Brasil. Rodriguésia 67: 489-531.

Riina, R., Carneiro-Torres, D.S., Peirson, J.A., Berry, P.E. \& Cordeiro, I. 2014. Further support for the Crotoneae Phylogeny: a new species of Brasiliocroton (Euphorbiaceae) based on morphological, geographical, and molecular evidence. Systematic Botany 39: 227-234.

Silva, O.L.M. \& Cordeiro, I. 2020. Taxonomic revision of Astraea Klotzsch (Euphorbiaceae). Plant Systematics and Evolution 306: 41.

Silva, O.L.M., Riina, R. \& Cordeiro, I. 2020. Phylogeny and biogeography of Astraea with new insights into the evolutionary history of Crotoneae (Euphorbiaceae). Molecular Phylogenetics and Evolution 145: 106738.

Webster, G.L. 2014. Euphorbiaceae. In: K. Kubitzki (Ed.), The Families and Genera of Vascular Plants vol. 11. SpringerVerlag, Berlin, p. 51-216.

Wurdack, K.J., Hoffmann, P. \& Chase, M.W. 2005. Molecular phylogenetic analysis of uniovulate Euphorbiaceae (Euphorbiaceae sensu stricto) using plastid $r b c L$ and $t r n L-F$ DNA sequences. American Journal of Botany 92: 1397-1420.

\section{Lista de Exsicatas}

Adorno, H. 61 (3); Almeida, J. 83 (3); Alunos de Botânica III ALCB 67204, CEPEC 107635 (4); Alves, L.J. 71 (3), 430 (7); Alves, M. 47 (3), EBNN 1144 (7); Amorim, A.M.A. 496, 6768 (3); Amorim, E. ALCB 21290 (3); Arbo, M.M. 5809 (4), 7172,7173 (3); Arouck-Ferreira, J.D.C. 364 (4); Atkins, S. PCD4594 (3); Bacelar, T.G. 55 (2); Bandeira, F.P. 167 (7); Barbosa, E. 2745 (7); Baumgratz, J.F. 139 (3); Bautista, H.P. 481, 535 (3), 3029 (4); Belém, R.P. 291 (3), 534 (2), 2062 (3); Benko-Iseppon, A.M. 1791 (4); Blanchet, J.S. 92, 167, 422 (1), 1071 (3), 3155 (5), 3216, 3216A (3), 3654 (4), 3155A (5), s.n. BM (7), G (1); Braga, D.V. IPA 73896 (7); Brito, J.C. 23, 96 (4); C.M.M. 18 (2); Cardoso, D. 801 (4), 2158 (3); Carneiro, D.S. 7, 8 (7), 62 (4); Carneiro, J. 1155 (3); Carneiro-Torres, D.S. 247 (4), 493 (7), 599 (4), 626 (7); Carvalho, A.M. 123 (2); Carvalho, A.M.V. 927, 1628 (3), 2005 (5), 6905, PCD2168 (4); Chautems, A. 306 (3); Conceição, A.A. 180 (3), 1069 (6), 1070 (3), 1364, 1679 (4); Conceição, A.S. 155 (7); Conceição, S.F. 302 (4), 577 (7); Coradin, J. 6194,6503 (4); Cordeiro, I. 3235, 3244, 3245 (3), 3386 (4), 3550 (3), 3554 (2); Cordeiro, J. 3727 (7); Correia, C. 416 (7); Costa, A.L. 501 , 798 (3), s.n. ALCB 2067 (7), ALCB 2068 (1), ALCB 2069 (7), ALCB 2070, ALCB 2097, ALCB 2137 (3), ALCB 2141 (4); Costa, G. 2142 (3), 2252 (4); Costa, H.V. UESC 16899 (2); Costa, J. 138 (7), 158 (3), PCD1769 (4); Duarte, A.P. 5948 (3), 6849 (7), 9356 (4); Ferraz RB 77752 (1); Ferreira, J.L. 279 (7); Ferreira, M.C. 165 (7), 639 (3); Ferreira, M.S.G. 250 (7); Fiaschi, P. 243, 1103 (3); Figueiroa, L.E. 46 (3); Filardi, F.L.R. 824 
(3); França, F. 957 (1), 6178 (7); Freire-Fierros, A. 1688 (4); Fróes, R.L. 20226 (4); Funch, R. 148, FCD5 (4); Furlan, A. CFCR 273 , CFCR 396, CFCR 7640 (4); Ganev, W. 60, 375 (6), 1943 (4); Giulietti, A.M. 2239 (6), CFCR 1424 (4); Glocker, E.F. 58 (1); Gomes, F.S. 852 (3); Gonçalves, L.M.C. 167 (4); Grupo Pedra do Cavalo 115, 284, 1065 (7); Guedes, M.L. 1192 (3), 1556 (4), 3170, 4071, 4713, 4878, 6036, 6533 (3), 7426 (4), 8199, 8212, 9605, 9615, 9974, 10818, 11105 (3), 11485, 12243, 14704 (7), 15138 (3), 15764, 16281 (7), 19023 (2), 19345, 20240 (3), 20655, 20913 (7), 21145, 23230 (3), 23895 (4), 24296, 24737 (7), ALCB 15832, ALCB 27521, MBM 249968 (3), ALCB 27934 (1), PCD 595, PCD 597, 1093 (4); Gusmão, E.F. 38 (7); Hage, J.L. 455, 917, 929 (2), 1118 (1), 1986, 2138 (2); Harley, R.M. 17215 , 17592, 18293 (3), 20545, 20581, 20640 (4), 22192 (3), 22442, 22681, 22723, 24417, 25311, 26231, 26625, 27194 (4), 27295 (6); Hatschbach, G. 39689,42375 (4), 47053 (3), 48011 (4), 53490 (3), 56805 (4), 63096, 63333 (3), 71273 (7), 75110, 75307 (3), 75396 (1), 75573 (7); Heringer, E.P. 3435a (1); Hurbath, F. 451 (2), 531, 620 (7), 666 (4); Jesus, J.A. 335 (2), 385, 485, 498, 563 (3); Jesus, N.G. 862 (7), 934 , 1788, 1828 (3), ALCB 86823, HRB 56593, HUEFS 144763 (1); Kawall, S.L. SP 468426, SPF 70593 (4); Lewis, G.P. 890, CFCR 7149, CFCR 7299 (4); Lima, A.F.S. UEC 183691 (3); Lima, L.R. 173 (4), 261 (6); Lombardi, J.A. 7145 (7), 8870 (3), 8912 (2); Longa, C.M. 12 (3); Loureiro, D.M. 465 (4); Luceño, M EBBN 478 (4); Machado, R.F. 104 (2), 487 (7); Maia, M.L. 1 (7); Marquete, R. 4434 (4); Martinelli, G. 5306, 5311 (4); Martins, D. ALCB 55227, ALCB 55228 (4); Matos, E.N. 701 (3); Mattos-Silva, L.A. 797, 831 (3), 3337,3386 (2), 3795 , 3925, 4153, 4187, 4584 (3); Mello-Filho, L.E. 3052 (3); Mello-Silva, R. CFCR 7206 (4); Melo, E. 1542, 2063 (7), 2963 (4), 3906,7055 (7), PCD 1214, PCD 1285, PCD 1356 (4); Menezes, N.L. CFCR 384 (4); Miranda, A.M. 6218 (3); Monteiro, R.F. 642 (4); Moraes, M.V. 573 (3); Moraes, P.L.R. 2787, 2983 (4); Mori, S.A. 10411 (2), 10468, 10579, 10598 (3), 10988 (2), 12197 (7), 12966, 13540 (4), 13641,14055 (3), 14259 (4); Moruz, C.V.A. 104 (4); Moura, L.M. 48 (4); Nascimento, A.F.S. 190 (3); Neves, S.P.S. 31, 52, 91, 153, 175 (4); Noblick, L.R. 2141 (3), 2840 (4), 3706 (7), 4448 (3); Novais, J.S. 24 (2); Nunes, T.S. 1354, 1414 (3); Oliveira, M. 2791 (7); Oliveira, M.V.M. 713 (7); Oliveira, P.P. 19 (3); Oliveira, R.P. 1993 (3); Orlandi, R. PCD 281 (4); Paraguassú, L. nov/92 (7); Paula-Souza, J. 5472 (7), 6053 (3), 6346, 9349, 9838 (7); Pereira, E. 2223 (4); Pereira, O.J. 5377 (3); Pinto, G.C.P. 133 (7), 54-58 (3), 160/83 (7), MBML 2473, SP 274822 (1); Pirani, J.R. 2758 (3), 2938 (5); Popovkin, A.V. 547 (7); Prance, G.T. 58303 (2); Queiroz, E. HRB 30896, RB 482298 (7); Queiroz, E.P. 1400 (3), 1473 (1), 1588 (3), 2173 (1); Queiroz, L.P. 1900 (4), 3187 (7), 5161, 5179, 7434 (4), 9121 (7), 9926 (4); Ribeiro, T. 209 (4); Rigueira, D. ALCB 67973, SPF 174075 (3); Rocha, A. IAC 23271 (7); Rodrigues, A.A.C. 2 (7); Roque, N. 709 (7), 1039, 4385, ALCB 61410 (4); s.col. K 1210207, K 1210208 (1); Sá, B.L. 106, 107 (7); Sant'Ana, S.C. 1108 (7), 1114, 1115 (3), 1123 (5), 1297 (3); Santana, W. ALCB 17947, SP 286809 (4); Santos, B.T.C. 476 (2); Santos, E.B. 49 (2); Santos, J.S. 85 (3); Santos, R.B. 45 (4); Santos, T.S. 409 (2), 608 (1), 704 (3), 856 (1), 4423 (2); Senna, L.R. 157 (4); Silva, G.C.A. 2 (3); Silva, M.I.B. 3 (3); Silva, M.M. 370 , 503 (3); Silva, N.T. 58303 (2); Silva, O.L.M. 147, 149 (7), 180, 181, 182, 183, 184, 185, 188 (4), 195 (3), 214 (7), 215, 216 (3), 217, 218 (7), 220 (3), 222, 223, 224, 225 (2), 227 (3), 228 (1), 229 (3), 230, 231, 232 (7); Silva-Castro, M.M. 1090, 1092 (6); Sobral, M. 7601 (4); Soeiro, R. 07/89 (3), $38 / 89$ (7), $43 / 89$ (3); Souza, R.S. 6 (7); Souza, V.C. 26557 (7); Souza-Silva, R.F. 156 (7); Stannard, B.L. H51838 (4); Thomas, W.W. 9709 (5), 9832, 10070 , 13081 (3), 14137 (1), 10060A (3); Turmas do $2^{\mathbf{0}}$ ano A/C 54 (3); Valadão, R.M. 9 (3); Vasconcelos, L.V. 609 (4); Vieira, L.P. 53 (7); Wawra, H. 342 (3); Webster, G.L. 25028 (7), 25066, 25076,25127 (3), 25769 (4), 25854 (3). 